\title{
Investigação sobre a Produção de Sinais em LIBRAS: uma Revisão Sistemática de Literatura em Glossários Técnicos
}

\author{
Rafael Pinto Granada, PPGEC/FURG, rafaelgranada@furg.br, \\ https://orcid.org/0000-0001-8359-2352 \\ Regina Barwaldt, C3/FURG, reginabarwaldt@furg.br, \\ https://orcid.org/0000-0002-0382-3892 \\ Luiz Oscar Homann de Topin, PPGMC/FURG, ltopin@gmail.com, \\ https://orcid.org/000-0002-6083-8302
}

\begin{abstract}
Resumo: O artigo tem como objetivo apresentar uma revisão sistemática para investigar as tecnologias utilizadas na implementação de glossários técnicos, assim como os critérios de acessibilidade e os métodos para elaboração de sinais em LIBRAS. A pesquisa foi realizada a partir da análise das publicações realizadas num período de 4 anos nas bases de dados: ACM, Redalyc, IEEE Xplore, BDTD, Portais de periódicos CAPES, SBIE e UFSC. Como resultado emergiu-se uma amostra final de 23 trabalhos, a qual indicou que as implementações em glossários técnicos para surdos necessitam de mais estudos, principalmente sobre o uso de novas tecnologias, recursos de acessibilidade alternativos como animações em línguas de sinais ou utilização de datilologia na forma escrita, além do emprego de fichas terminológicas para registro dos sinais produzidos.
\end{abstract}

Palavras-chave: glossários técnicos, acessibilidade, línguas de sinais, surdos.

\section{Investigation on the Production of Signs in LIBRAS: a Systematic Review of the Literature in Technical Glossaries}

\begin{abstract}
The paper aims to present a systematic review to investigate the technologies used in the implementation of Technical Glossaries, as well as the accessibility criteria and methods for elaborating signs in LIBRAS. The research was carried out from the analysis of publications made over a period of 4 years in the databases: ACM, Redalyc, IEEE Xplore, BDTD, CAPES, SBIE and UFSC journals. As a result, a final sample of 23 works emerged, which indicated that the implementations in technical glossaries for the deaf require further studies, mainly on the use of new technologies, alternative accessibility resources such as sign language animations or use of fingerspelling in written form, in addition the use of terminological cards for recording the produced signs.
\end{abstract}

Keywords: technical glossaries, accessibility, sign languages, deaf.

\section{Introdução}

O reconhecimento da Língua Brasileira de Sinais (LIBRAS) como meio legal de comunicação por meio da Lei $10.436^{1}$ assegurou o direito de expressão dos surdos nos

\footnotetext{
${ }^{1}$ Dispõe sobre a Língua Brasileira de Sinais - Libras e outras providências. Acessível pela URL: https://www.planalto.gov.br/ccivil_03/leis/2002/L10436.htm. 
diversos espaços da sociedade. Outra conquista foi o sancionamento da Lei $13.409^{2}$ que inclui pessoas com deficiência no sistema de cotas adotado pelas universidades e em escolas de ensino técnico da esfera federal.

Dessarte, aos surdos que alcançam os cursos de nível técnico e superior enfrentam o problema nas disciplinas que possuem termos complexos, adequados às suas especificidades, sem tradução em língua de sinais, prejudicando seu aprendizado (MARTINS, 2016). A carência de sinais para esses termos pode acontecer em função da LIBRAS ser uma língua ainda em construção (GRANADA, 2019).

Dessa maneira, os surdos recorrem a materiais didáticos em línguas de sinais (LS) para suprir a necessidade de aprender termos específicos e, normalmente utilizam a Internet para acessar os repositórios de sinais mais conhecidos como glossários técnicos. Assim, nas mudanças culturais e tecnológicas, como a procura por ingresso nas instituições de ensino, sobrevém a necessidade de elaborar mais sinais em LIBRAS (SILVA, 2011).

Estes glossários surgiram com o objetivo de prover sinais para expressar termos específicos nas inúmeras áreas de formação educacional e profissional (MALACARNE, 2018; MCKEOWN, 2019).

Em relação à interface gráfica apresentados por esses softwares, pode se observar uma falta de padronização, ou seja, desenvolvidos de forma empírica, estruturados com base na linguagem escrita sem suporte para línguas de sinais e recursos visuais adequados, caracterizando um problema de acessibilidade para os surdos ao utilizarem tais artefatos digitais. Nesse contexto, acessibilidade é o requisito essencial para um indivíduo independente de sua condição física ou mental possa interagir um ambiente computacional, com intuito de potencializar seu processo de aprendizagem (VIANNA, 2019).

Diante do exposto, realizou-se uma revisão sistemática de literatura com o objetivo de investigar as tecnologias utilizadas na implementação de glossários técnicos, os critérios de acessibilidade e os métodos para elaboração de novos sinais.

\section{Metodologia}

O mapeamento sistemático baseou-se no processo descrito por Kitchenham (2007) que propõe uma metodologia constituída por 3 fases: planejamento, condução e resultados. São apresentadas duas formas de resultados, a primeira é uma revisão sistemática da literatura e a outra, um mapeamento sistêmico. Todavia, neste trabalho será considerado somente a primeira opção, apenas fontes atualizadas de dados.

\subsection{Fase de Planejamento}

Essa fase é composta por 4 etapas:

1. Questão principal de pesquisa e questões secundárias;

2. Delimitação do método de pesquisa;

3. Seleção dos estudos (artigos, periódicos, dissertações ou teses);

4. Extração de dados.

\footnotetext{
${ }^{2}$ Dispõe sobre a reserva de vagas para pessoas com deficiência nos cursos técnico de nível médio e superior das instituições federais de ensino. Para mais informações, acesse: https://www.planalto.gov.br/ccivil_03/_ato2015-2018/2016/lei/113409.htm. 


\subsubsection{Questão principal de pesquisa e questões secundárias}

Considerando que as indagações da pesquisa devem refletir os objetivos da revisão de literatura, apresentamos a questão principal e as secundárias conforme Quadro 1:

Quadro 1. Questões da pesquisa

\begin{tabular}{|c|l|l|}
\hline \multirow{2}{*}{$\begin{array}{c}\text { Tipo } \\
\text { Principal }\end{array}$} & \multicolumn{2}{c}{ Quais tecnologias, plataformas e critérios de acessibilidade são utilizados para glossários } \\
& técnicos, assim como os procedimentos adotados para elaboração de sinais?
\end{tabular}
Fonte: elaborado pelos autores

Apresentadas as questões de pesquisa, na sequência, será demonstrada a etapa de delimitação do método de pesquisa.

\subsubsection{Delimitação do método de pesquisa}

Esta etapa apresenta os critérios utilizados na pesquisa, isto é, os idiomas compreendidos, as bases de dados a serem consultadas, as palavras-chave, técnicas de busca, e período de abrangência. As bases de dados selecionadas mais pertinentes e que possuem relação direta com tema são os anais de eventos, periódicos, teses, dissertações e revistas científicas com publicações na área da educação e/ou computação. Desse modo, escolheu-se 4 bases de dados nacionais e 3 internacionais, totalizando 7 bases consultadas, as quais são apresentadas conforme Quadro 2:

Quadro 2. Bases de dados Nacionais e Internacionais

\begin{tabular}{|c|c|}
\hline Bases de Dados Nacionais & Bases de Dados Internacionais \\
\hline $\begin{array}{c}\text { Biblioteca Digital Brasileira de Teses e } \\
\text { Dissertações (BDTD) }\end{array}$ & ACM Digital Library \\
\hline Portal de Periódicos da CAPES/MEC & IEEE Xplore Digital Library \\
\hline Portal do Simpósio Brasileiro de & Sistema de Información Científica \\
Informática na Educação (SBIE) & Redalyc \\
\hline Portal de Periódicos da UFSC & \\
\hline
\end{tabular}

Fonte: elaborado pelos autores

Para a realização das buscas nas bases de dados científicas definiu-se as Línguas Inglesa e Portuguesa com um intervalo de tempo entre 2016 e 2019, totalizando 4 anos de pesquisas. Assim, utilizou-se as palavras-chave sinais, glossário, surdos, interface e acessibilidade, bem como, seus equivalentes na Língua Inglesa, signs, glossary, deaf, interface e accessibility. Empregou-se também sinônimos como dicionário e design, além de conectores lógicos ( $A N D$ e OR) com o objetivo de potencializar o processo de busca. Na sequência são apresentadas as 6 strings que melhor se adequaram para encontrar trabalhos relacionados relevantes:

1. (Sinais AND glossário AND surdos);

2. (Sinais AND glossário OR surdos) AND interfaces AND acessibilidade;

3. (Sinais AND dicionário AND surdos);

4. (Design AND glossário AND surdos);

5. (Glossário OR Dicionário) AND acessibilidade;

6. (Glossário OR Dicionário) AND interfaces. 


\subsubsection{Seleção dos estudos}

Esta etapa consiste na aplicação das strings de busca nas bases de dados científica mencionadas anteriormente e o resultado é apresentado no Quadro 3.

Quadro 3. Resultado preliminar da busca nas Bases de dados

\begin{tabular}{|l|c|}
\hline Bases de Dados Nacionais & No de Estudos \\
\hline Biblioteca Digital Brasileira de Teses e Dissertações (BDTD) & 12 \\
\hline Portal de Periódicos da CAPES/MEC & 7 \\
\hline Portal do Simpósio Brasileiro de Informática na Educação (SBIE) & 1 \\
\hline Portal de Periódicos UFSC & 6 \\
\hline Bases de Dados Internacionais & \\
\hline ACM Digital Library & 9 \\
\hline IEEE Xplore Digital Library & 5 \\
\hline Sistema de Información Científica Redalyc & 2 \\
\hline \multicolumn{2}{|r|}{ Fonte: elaborado pelos autores } \\
\hline
\end{tabular}

Encontrou-se o total de 42 trabalhos, sendo que 26 foram em bases de dados nacionais e os restantes em bases internacionais. Para realizar a seleção dos estudos primários dessa RSL e garantir a precisão sobre o tema escolhido definiu-se critérios de inclusão e exclusão, que são apresentados no Quadro 4.

Quadro 4. Critérios de Inclusão e Exclusão

\begin{tabular}{|c|c|}
\hline Critérios de Inclusão & Critérios de Exclusão \\
\hline $\begin{array}{l}\text { Implementação de glossários em } \\
\text { línguas de sinais }\end{array}$ & $\begin{array}{l}\text { Estudos que não respondam as questões } \\
\text { desta RSL }\end{array}$ \\
\hline Produção de sinais & $\begin{array}{l}\text { Os artigos redundantes, incompletos, } \\
\text { duplicados ou pagos }\end{array}$ \\
\hline Interfaces de glossários técnicos & Sem autoria \\
\hline Acessibilidade em glossários técnicos & $\begin{array}{l}\text { Artigos em outros idiomas que não sejam } \\
\text { o Inglês ou Português }\end{array}$ \\
\hline
\end{tabular}

A etapa seguinte refere-se à busca de trabalhos com a utilização dos critérios de exclusão e inclusão, sendo assim, a seleção final teve como resultado o total de 23 estudos, 16 nacionais e 7 internacionais, conforme Quadro 5.

\section{Quadro 5. Resultado final da busca nas Bases de Dados}

\begin{tabular}{|l|c|}
\hline Bases de Dados Nacionais & N $^{\mathbf{0}}$ de Estudos \\
\hline Biblioteca Digital Brasileira de Teses e Dissertações (BDTD) & 10 \\
\hline Portal de Periódicos da CAPES/MEC & 2 \\
\hline Portal do Simpósio Brasileiro de Informática na Educação (SBIE) & 1 \\
\hline Portal de Periódicos UFSC & 3 \\
\hline Bases de Dados Internacionais & \\
\hline ACM Digital Library & 3 \\
\hline IEEE Xplore Digital Library & 3 \\
\hline Sistema de Información Científica Redalyc & 1 \\
\hline
\end{tabular}

Fonte: elaborado pelos autores

\subsubsection{Extração dos dados}

O processo de extração de dados consiste em prover as respostas para as questões secundárias que compõem a pesquisa. Essa técnica assegura a execução dos mesmos critérios de dados no processo de extração em todos os documentos, auxiliando na sua classificação (BELTRAN, 2017). O Quadro 6 apresenta os dados obtidos por meio da extração dos dados: 
Quadro 6. Dados obtidos pela técnica de extração

\begin{tabular}{|c|c|}
\hline \multicolumn{2}{|l|}{ Questão Secundária 1} \\
\hline Tecnologias utilizadas & Resultados \\
\hline Ajax & 1 \\
\hline Android & 1 \\
\hline Bootstrap & 2 \\
\hline HTML5 & 8 \\
\hline$M y S Q L$ & 3 \\
\hline PHP & 2 \\
\hline PowerPoint & 2 \\
\hline Python & 1 \\
\hline Youtube & 2 \\
\hline \multicolumn{2}{|l|}{ Questão Secundária 2} \\
\hline Plataformas utilizadas & Resultados \\
\hline Dispositivos Móveis & 5 \\
\hline Software & 4 \\
\hline Web & 10 \\
\hline Outros Formatos & 4 \\
\hline \multicolumn{2}{|l|}{ Questão Secundária 3} \\
\hline Critérios de acessibilidade & Resultados \\
\hline Vídeos com legendas & 4 \\
\hline Vídeos em línguas de sinais (LS) & 10 \\
\hline Textos curtos e objetivos & 6 \\
\hline Imagens explicativas & 10 \\
\hline Presença de avatar & 5 \\
\hline Recursos explanatórios complementares & 13 \\
\hline Animações em LS & 1 \\
\hline Uso de datilologia escrita & 1 \\
\hline \multicolumn{2}{|l|}{ Questão Secundária 4} \\
\hline Procedimentos para elaboração de sinais & Resultados \\
\hline Escolha de especialistas na produção de sinais & 23 \\
\hline Seleção dos termos na língua oral & 23 \\
\hline Elaboração dos sinais para os termos escolhidos & 23 \\
\hline Gravação dos sinais & 19 \\
\hline Validação pela comunidade surda & 23 \\
\hline Publicação em um glossário técnico & 19 \\
\hline Uso de ficha terminológica para registro dos sinais & 13 \\
\hline
\end{tabular}

Fonte: elaborado pelos autores.

Dos estudos escolhidos somente alguns apresentaram respostas para a pergunta QS1 - "Quais tecnologias são utilizadas para implementar glossários técnicos?". Encontrou-se bancos de dados, Youtube e linguagens de programação web. Entretanto, a mais utilizada é a linguagem HTML5, presente em 8 estudos seguida pelo MySQL, com 3 trabalhos, o que mostra a preferência dos pesquisadores no uso dessas tecnologias para o desenvolvimento de glossários técnicos para surdos.

Quanto à QS2 - "Os glossários técnicos são desenvolvidos em quais plataformas?", apresentou o predomínio das plataformas web dentre os estudos selecionados com um total de 10 incidências, seguidos pelos dispositivos móveis com 5 ocorrências. Isso demonstra a popularização do uso da web e aplicativos de celular pelo público-alvo, pois a utilização dessas plataformas são recursos facilitadores para construção de conhecimento, interação social, autonomia, entre outros (NOGUEIRA, 2018). Nesse sentido, com a facilidade de acesso a essas plataformas encontrou-se um número reduzido de estudos glossários em softwares e outros formatos (Livros, CDs, DVD), com 4 incidências. 
A questão QS3 - "Quais critérios de acessibilidade são considerados? ”, foram escolhidos os princípios de acessibilidade utilizados para artefatos digitais da Internet citados por Carmo (2019) e Vianna (2019), os quais são apresentados no Quadro 6. Como trata-se de glossários técnicos para surdos, é usual que a maioria dos estudos apresentem vídeos em LS, imagens explicativas e recursos explanatórios complementares para os sinais apresentados. Dessa forma, o critério "recursos explanatórios" obteve incidências em 13 estudos enquanto as outras apresentaram 10 ocorrências.

No entanto, dentre os 23 estudos selecionados, somente 1 trabalho apresentou interface com animações em línguas de sinais, recurso que facilita a utilização por ser intuitiva, ou seja, retira a necessidade do usuário surdo em interpretar textos enquanto navega por um glossário. Outro fator interessante foi a carência na utilização de recursos alternativos à elementos textuais de línguas orais, tais como o uso de datilologia ${ }^{3}$ na forma escrita, que apresentou somente uma ocorrência.

A questão QS4 - "Quais os procedimentos foram utilizados para elaborar sinais em glossários técnicos?", normalmente, a elaboração de novos sinais possui um determinado padrão estrutural, utilizados pela maioria dos estudos selecionados, apresentados no Quadro 6. Os procedimentos "escolha de especialistas", "seleção de termos", "elaboração de sinais" e "validação" apareceram em todos os trabalhos escolhidos. Por outro lado, o "uso de ficha terminológica para registro de sinais" obteve apenas 13 incidências, que demonstrou pouca preocupação dos pesquisadores em relação à polissemia e sinonímia. A primeira refere-se a um sinal ter vários significados e a segunda, sobre dois sinais diferentes possuírem o mesmo sentido (JOHSTON, 2007). Contudo, ficha terminológica é um documento estruturado que deve conter todas as informações sobre cada termo (sinal) e é relevante para o seu registro em dicionários ou glossários (FAULSTICH, 2014).

\subsection{Condução}

Ao executar o protocolo de revisão, são gerados resultados preliminares os quais serão escolhidos conforme os critérios de inclusão definidos, concordando com Neves (2019). Nesta fase, podem ser encontrados estudos publicados em várias fontes científicas e, assim, fez-se necessário realizar a seleção apenas da versão mais completa do estudo (BELTRAN, 2017).

\subsection{Resultados}

Essa etapa consiste em complementar o conteúdo apresentado nos quadros anteriores, propiciando uma melhor compreensão e visualização dos dados, além de apresentar novas informações. A Figura 1 apresenta a quantidade de estudos escolhidos nos repositórios de dados selecionados sem a utilização dos critérios de inclusão/exclusão. Nessa busca, o maior número de trabalhos se encontra nas bases de dados da BDTD seguido pela ACM Digital.

\footnotetext{
${ }_{3}^{3}$ Datilologia é a soletração de uma palavra nas línguas de sinais. É possível utilizá-la na forma escrita, como um texto, mas é necessários softwares de terceiros.
} 
Figura 1. $\mathbf{N}^{\circ}$ de estudos encontrados sem critérios de inclusão/exclusão

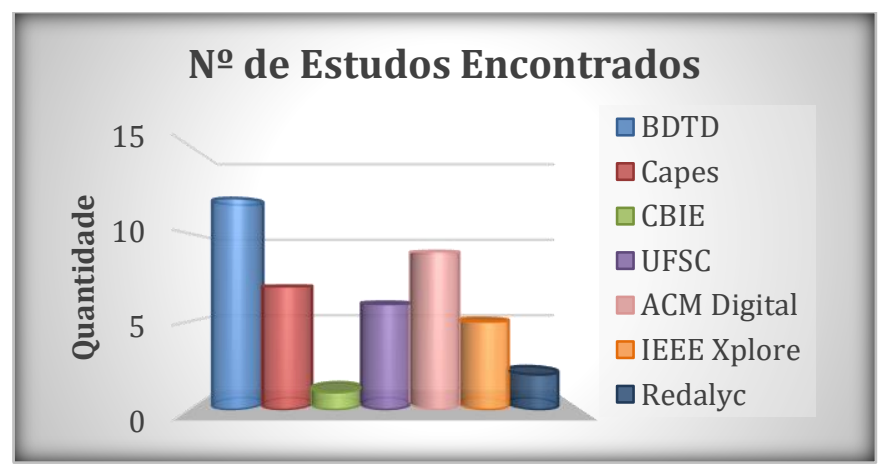

Fonte: elaborado pelos autores

A Figura 2 apresenta os estudos selecionados após empregar os critérios de inclusão/exclusão. A base de dados BDTD apresentou 44\% dos trabalhos pertinentes a esta revisão sistemática de literatura, seguida pela ACM Digital, IEEE Xplore e Portal da UFSC com 13\% dos trabalhos. Dos 23 estudos definidos, 3 são de autoria do próprio autor e encontram-se em 2 bases de dados diferentes. Isso representa $13 \%$ dos estudos escolhidos e demonstra a relevância de sua pesquisa sobre glossários técnicos.

Figura 2. $\mathbf{N}^{0}$ de estudos selecionados com critérios de inclusão/exclusão

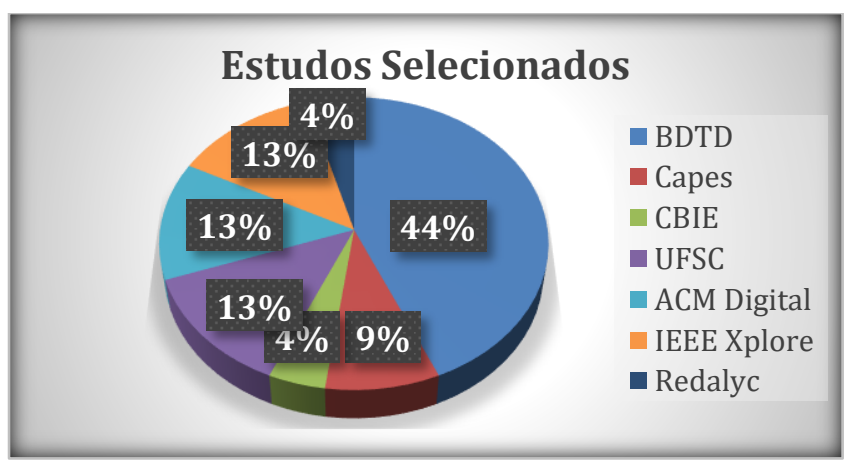

Fonte: elaborado pelos autores

A Figura 3 contempla a pergunta QS1, ou seja, representa as tecnologias utilizadas para o desenvolvimento dos glossários em línguas de sinais, que foram encontradas nos 23 trabalhos selecionados. A pesquisa indicou a linguagem de programação HTML5 como a tecnologia mais utilizada nos estudos selecionados, seguido pelo Banco de Dados MySQL.

Figura 3. Tecnologias utilizadas em glossários técnicos

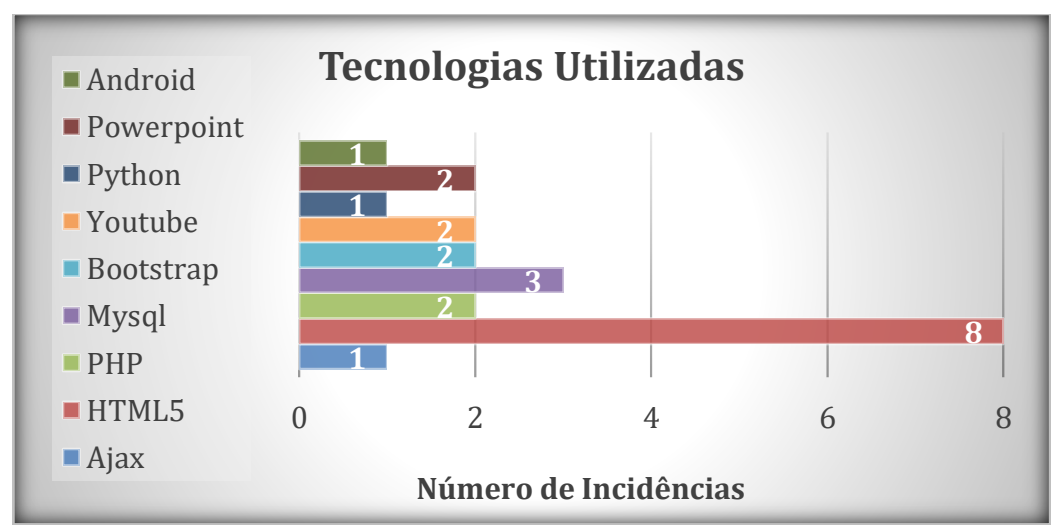

Fonte: elaborado pelos autores 
A Figura 4 refere-se à QS2, que representa em quais plataformas os glossários estão sendo desenvolvidos. Atualmente, pela facilidade de uso e disponibilidade da Internet, a plataforma web tem sido principal meio de acesso aos glossários técnicos. Aparece em $44 \%$ dos glossários apresentados nos estudos selecionados, acompanhado pelos dispositivos móveis com $22 \%$ e, por fim, softwares e outros formatos com $17 \%$.

Figura 4. Plataformas utilizadas pelas interfaces de glossários

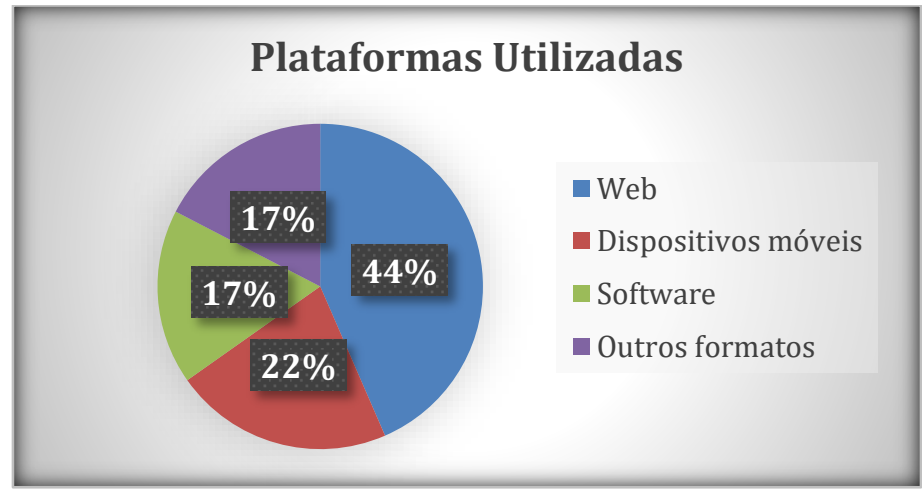

Fonte: elaborado pelos autores

A QS3 é apresentada por meio da Figura 5, que demonstra os critérios de acessibilidade utilizados pelos glossários encontrados nos estudos selecionados. Pode-se analisar que os pesquisadores além de apresentarem os sinais nos glossários, preocuparam-se em torná-los de fácil entendimento para o público-alvo, o que indica a maior incidência dos recursos explanatórios, vídeos em LS e imagens explicativas. Por outro lado, não utilizaram recursos que facilitariam a acessibilidade e navegabilidade do glossário de uma forma geral como animações em LS, uso de datilologia escrita e textos curtos.

Figura 5. Critérios de acessibilidade encontrados nos estudos selecionados

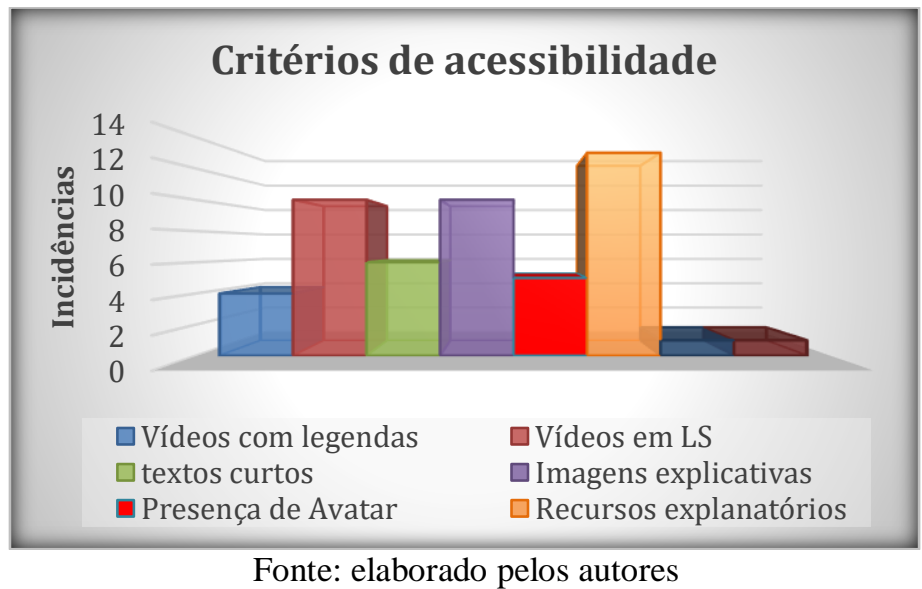

A Figura 6 contempla a QS4 que apresenta alguns procedimentos relevantes para elaboração de sinais. Nessa figura, dos 23 estudos analisados podemos perceber que nenhum realizou os estágios para produção de novos sinais em sua totalidade, porém consideraram primordial a escolha de especialistas, seleção de termos e validação. Quatro trabalhos não publicaram os sinais em glossários, disponibilizando-os no formato impresso e, assim, não fez-se necessário realizar o processo de filmagem. Dessa forma, os procedimentos "publicação em glossário técnico" e gravação de sinais atingiram 19 incidências nesta revisão sistemática. 
Figura 6. Procedimentos para Produção de Sinais

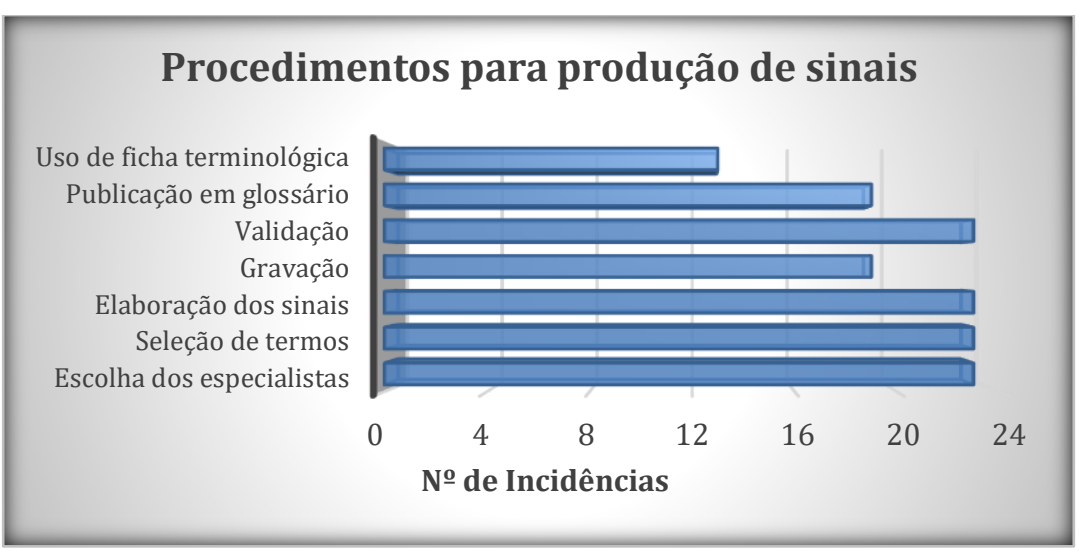

Fonte: elaborado pelos autores

A Figura 7 demonstra a disposição dos trabalhos publicados sobre glossários técnicos nos últimos anos. Considerou-se os trabalhos publicados anualmente desde 2016 até 2019. O ano inicial obteve duas publicações, seguido por 2017 com 5 e, finalmente, os anos 2018 e 2019 com 8 incidências cada um.

Figura 7. Resultados dos estudos por ano

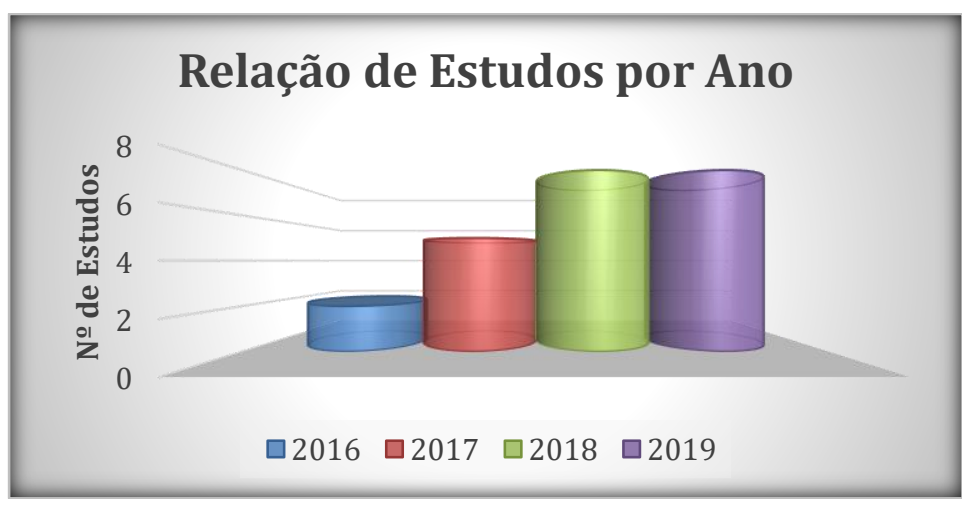

Fonte: elaborado pelos autores

Segundo as informações apresentadas acima, percebemos o interesse crescente da comunidade acadêmica sobre o desenvolvimento de glossários técnicos.

\section{Considerações Finais}

Este trabalho forneceu um panorama sobre o que está sendo implementado em glossários técnicos em línguas de sinais. Para tanto, realizou-se uma revisão sistemática, delineada por uma questão principal e quatro subquestões, que exploraram as tecnologias, plataformas, critérios de acessibilidade empregados e os procedimentos para produção de sinais.

De uma forma adventícia, ainda que existam tecnologias com maior aplicabilidade na programação de glossários técnicos, os pesquisadores possuem como preferência o HTML5 como linguagem de implementação e o uso da plataforma web, seguido pelos dispositivos móveis. Os artefatos digitais estudados atenderam parcialmente aos critérios de acessibilidade sugeridos e uma parcela considerável não utilizou recursos que facilitariam a interação dos surdos como animações em LS e uso de datilologia na forma escrita. Isso pode indicar que para seu desenvolvimento é preciso compreender com maior especificidade as necessidades desses usuários, pois ainda apresentam dificuldades em interagir com glossários projetados para ouvintes. 
O trabalho também apresentou os procedimentos empregados para a produção de sinais e observou-se que a maioria dos estudos selecionados realizaram as etapas necessárias para a constituição desse processo, exceto o uso de fichas terminológicas, mecanismo importante para o registro do sinal e controle da polissemia e sinonímia.

Destarte, como trabalho futuro sugere-se buscar estudos em outras bases de dados com um maior período de pesquisa, a fim de complementar a revisão sistemática apresentada, pois em virtude do número de estudos encontrados, percebeu-se que o tema abordado ainda é pouco explorado pela comunidade cientifica.

\section{Referências}

GRANADA, R. P. et al. Computational glossary in LIBRAS: an experience in undergraduate program of Information Systems. In: 2019 IEEE Frontiers in Education Conference (FIE), Cincinnati, USA, 2019, p, 1-5.

BELTRAN, P.; RODRIGUEZ, P.; CEDILLO, P. A Systematic Literature Review for Development, Implementation and Deployment of MOOCs Focused on Older People. In: 2017 (INCISCOS), Quito: IEEE, 2017, p. 287-294.

CARMO, G. M.; PAIVA, D. M.; CAGNIN, M. I. How to develop accessible web interfaces for deaf people?. In: Proceedings of the 18th Brazilian Symposium on Human Factors in Computing Systems, Vitória Espírito Santo Brazil: ACM, 2019, p. 1-10.

FAULSTICH, E. L. de J. Características conceituais que distinguem o que é de para que serve nas definições de terminologias científica e técnica. As ciências do léxico: lexicologia, lexicografia, terminologia, Vol. VII. Campo Grande, MS: Ed. UFMS, 2014.

JOHSTON, T.; SCHEMBRI, A.; Australian Sign Language: Auslan: An Introduction to Sign Language Linguistics. Cambridge University Press.1st Edition. 2007. 323 p.

KITCHENHAM, B.; CHARTERS, S. Guidelines for performing systematic literature reviews in software engineering version 2.3. Software Engineering Group, v. 45, n. 4, p. 1051, 2007.

MALACARNE, V; DE OLIVEIRA, V. A contribuição dos sinalários para a divulgação científica em Libras. Ensino em Re-vista, Uberlândia, v.25, n. 2, p. 289-305, set. 2018.

MCKEOWN, Caitlin, McKeown, Julia. Accessibility in Online Courses: Understanding the Deaf Learner. Springer Nature - TechTrends, Basel, Switzerland, v. 63, p. 506-513, 2019.

MARTINS, F.; STUMPF, M. Coleta e Registro de sinais-termos psicológicos para Glossário de Libras. Revista Leitura, Maceió, v.1, n. 57, p. 35-59, jun. 2016.

NEVES, D. F. et al. Governança Colaborativa em Instituições de Ensino: Uma Quasi-Revisão Sistemática da Literatura. Revista RENOTE, v. 17, n. 3, p. 21-30, 2019.

NOGUEIRA, C. M; CARNEIRO, M. N; SILVA, T. A. O uso social das tecnologias de comunicação pelo surdo: limites e possibilidades para o desenvolvimento da linguagem. Revista Pesquisa Qualitativa, São Paulo, v. 6, n. 12, p. 470, 2018.

SILVA, I. dos S.; SANTANA, B. P. Libras e Ensino Técnico: A necessidade de novos sinais. VII Jornada de Iniciação Científica - PIVIC Mackenzie/Mack Pesquisa. São Paulo, 2011.

VIANNA, A. B. A acessibilidade e a usabilidade nos ambientes virtuais de aprendizagem e o estudante surdo. São Paulo: USP, 2019. 257 p. Tese de Doutorado. 